\title{
STRUCTURAL OPTIMIZATION OF AEROFOILS FOR MANY CRITERIA
}

\author{
Schlieter T.*, Długosz A.**
}

\begin{abstract}
In this paper a problem of structural optimization of an aerofoil is examined. Authors' implementation of a multiobjective optimization algorithm based on differential evolution and elements of game theory is used as an optimization tool. Real requirements asked of aerofoil systems, described by mathematical functions are used as optimization objectives. Values of these functionals are determined numerically multiple times during the course of optimization. On the basis of a wing of unmanned aerial vehicle (UAV), a parametric numerical model is built. The inner geometry of the wing is described by 24 design variables and is subject to the optimization, while the outer geometry doesn't change.
\end{abstract}

\section{Keywords: Multiobjective optimization, Aerofoil, Differential evolution, Game theory, FEM.}

\section{Introduction}

In this research a novel multiobjective optimization algorithm was developed utilizing differential evolution and elements of game theory. Differential evolution is a single objective, population based optimizer (Storn, 1997). Game theory elements are used to compare solutions, assuming each player represents a single objective. The developed algorithm can be useful in a wide range of real world multiobjective problems, including optimization of composite, thermoelastic, electrostatic and piezoelectric structures. Aerofoil design optimization problem was presented as a numerical example of application of designed algorithm in mechanical systems. FEM software was used to determine values of objectives considered during the course of optimization.

Aim of this study is to find an optimal design or a set of Pareto-optimal designs of an inner geometry of an aerofoil system under mechanical loads representing working conditions. The optimization process is performed for many criteria at once and thus a multiobjective optimization approach is used. Main assumptions of the research include:

- Outer geometry of the aerofoil is not subject to the optimization task. Only the structural optimization of an inner geometry is performed and the outer shape is assumed to be fixed and thus no computational fluid dynamics (CFD) analyses are performed.

- Authors' implementation of multiobjective optimization algorithm based on differential evolution and elements of game theory is used.

- $\quad$ Parametric numerical model, with geometry based on a wing of a real unmanned aerial vehicle (UAV) is used as a numerical example.

- $\quad$ FEM software is adapted to solve boundary value problems in order to determine values of optimization functionals.

- $\quad$ Linear-elastic material models are assumed, isotropic and orthotropic for selected areas of the model (Gay, 2014), (Gibson, 2012).

Polyurethane foam is described with isotropic properties and the remaining materials with orthotropic properties. Distribution of materials in the wing is shown in Fig. 1.

* Eng. Tomasz Schlieter, MSc.: Department of Computational Mechanics and Engineering, Silesian University of Technology, Akademicka 2A, 44-100 Gliwice; PL, tomasz.schlieter@polsl.pl

** Assoc. Prof. Eng Adam Długosz, DSc.: Department of Computational Mechanics and Engineering, Silesian University of Technology, Akademicka 2A, 44-100 Gliwice; PL, adam.dlugosz@polsl.pl 


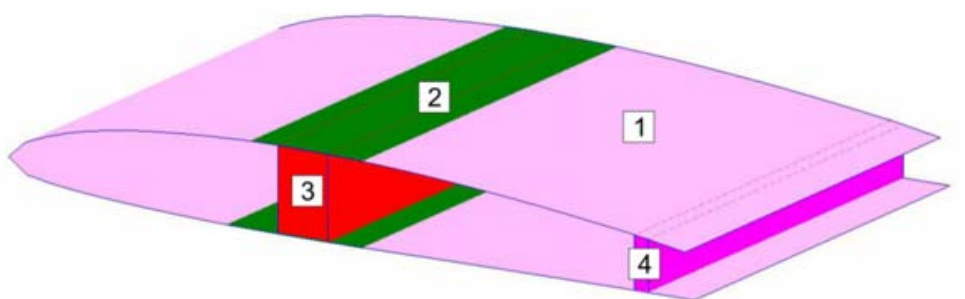

Fig. 1: Distribution of materials. 1 - carbon fabric $62 \mathrm{~g} / \mathrm{m}^{2}$, foam, glass fabric $24 \mathrm{~g} / \mathrm{m}^{2} ; 2$ - carbon fabric $62 \mathrm{~g} / \mathrm{m}^{2}, ; 3$ - carbon fabric $160 \mathrm{~g} / \mathrm{m}^{2} ; 4$ - glass fabric $24 \mathrm{~g} / \mathrm{m}^{2}$.

\section{Multiobjective optimization algorithm}

Most successful multiobjective optimization algorithms, using Pareto approach, such as NSGA-II, MOGA or SPEA2, utilize the methods of comparing feasible solutions within the population for effective optimization, for at most three criteria (Deb, 2002 and 1999). These include the following groups:

- methods, which refer to the number of individuals by which particular solution is dominated (dominance rank),

- methods, which refers to the number of individuals which particular solution dominates (dominance count),

- methods, where each of the individual is categorized on the basis of the membership to the separate fronts (dominance depth).

In order to solve practical optimization tasks for three or more criteria alternative approaches are applied. One of the proposals is a combination of game theory and single-objective heuristic algorithms, such as Differential Evolution.

The idea behind coupling DE and elements of game theory to solve multiobjective optimization problems comes down to treating $n_{o b j}$ objectives as players, playing a cooperative game, trying to improve their respective objectives with the resources given and sharing the information with each other, iteratively looking for a Nash equilibrium. Each player is given a part of design variable vector at random as their resources, while the rest of the vector is fixed and determined by other players' choices. Fixed values of design variables are taken from previous iteration of the algorithm, where different player made his choice in the process of optimization with regard to an objective assigned to him. To assure diversification of solutions after each player makes his move, using a single objective DE optimizer to find a proposed solution, the assignment of resources is changed in a way each design variable is modified by one and only one player.

The implementation of a resulting multiobjective optimization algorithm was developed by authors in C++ programming language. Communication with FEM software, which is utilized for calculation of fitness function values, is realized by script files allowing to run desired analyses on parametric model without the need of using Graphic User Interface (GUI) and report text files generated after running an analysis. Design variables are used as input data to generate Patran Command Language scripts and values of objectives are output data extracted from report files.

\section{Formulation of the problem}

Objectives in the optimization problem are devoted to minimization of maximal equivalent stresses, minimization of maximal displacements, minimization of total mass of the model and maximization of difference between values of modal frequency of a system and a given frequency - these are the examples of conditions required of aerofoil systems, although other criteria based on specific needs can be formulated. Values of functionals used as objectives are computed using FEM simulations. Boundary conditions and material properties are fixed during the optimization run and geometry of the model, described by design variables is changed to fit the declared needs:

$f_{1}$-minimization of the maximal value of the equivalent (von Mises) stress of an aerofoil under given load,

$f_{2}$-minimization of the maximal translational displacement of an aerofoil under given load,

$f_{3}$ - maximization of the minimal difference between any of first 10 modal frequencies of an aerofoil and a reference frequency $f_{\mathrm{r}}=30 \mathrm{~Hz}$, 
$f_{4}$ - minimization of the total mass of an aerofoil.

Values of aforementioned functionals are calculated numerically by means of FEM in MSC Patran/Nastran software based on a parametric model. Functionals $f_{1}$ and $f_{2}$ are calculated by running a static structural analysis under a given load. Functional $f_{3}$ is calculated by running a modal analysis. Problem of maximization of $f_{3}$ is transformed to a minimization problem by multiplying a fitness function value by 1 . Functional $f_{4}$ is calculated on the basis of geometry of the model and does not require solving a boundary-value problem.

\section{Numerical model}

Example of parametric numerical was build based on real structure of an UAV wing (Fig. 2). Approximate dimensions of the structure are: total length $\sim 1800 \mathrm{~mm}$ and transverse direction $\sim 200 \mathrm{~mm}$.

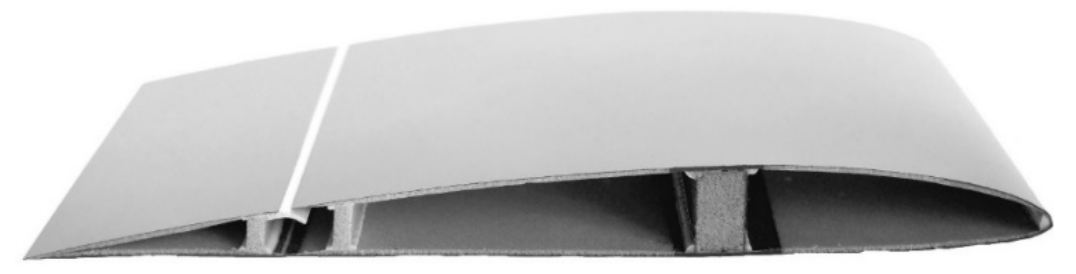

Fig. 2: Real structure of a UAV wing on which the numerical model was based.

Wing consists of two parts: centerwing and end part. The aileron is not included in the numerical model as its effect on the load transfer is negligible. Three characteristic cross sections of a wing can be distinguished: end cross section, middle cross section and base cross section. These geometries are controlled by 24 design variables, defining the inner shape of the wing and are described in Fig. 3.

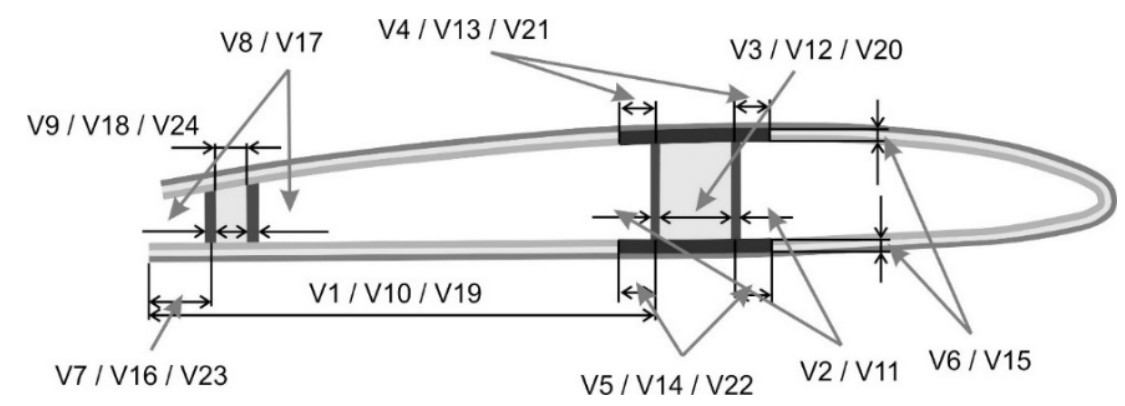

Fig. 3: Design variables controlling the inner shape of the wing.

The model is discretized using hex 8 and quad4 elements, and the resulting mesh consists of about $\sim 21500$ nodes and $\sim 19000$ elements, depending on the shape of an inner structure. Simplified boundary conditions applied in the numerical model include a fix in all degrees of freedom on one side of the model and an evenly distributed total load $\mathrm{P}=5 \mathrm{kN}$ on the bottom surface. Boundary conditions are shown in the Fig. 4 with the geometry flipped upside down.

\section{Results and conclusions}

Results of this research were obtained after solving approximately 25000 boundary value problems and consist of a set of 249 Pareto-optimal (non-dominated) solutions found during the course of optimization. Values of objective functions for the obtained solutions are shown in the figures in two ways. A $3 \mathrm{D}$ plot with values of the $f_{1}$ function represented by colour, and the remaining fitness function as values on the axes is shown in the Fig. 5.

Multiobjective optimization of aerofoil systems have been presented. Optimization of the parameters for the aerofoil have been solved for four different proposed functionals. More functionals related to particular needs asked of aerofoil systems can be formulated. New designs better than the existing design were found for each optimized objective. Stress and displacement values are acceptable even for the lightest structure 
found. No sweet spots were found in the solution space, which was expected due to the contradictory nature of optimized objectives. Application of presented method of optimization of an aerofoil system can significantly enhance the process of its design with respect to the many requirements asked of such systems.

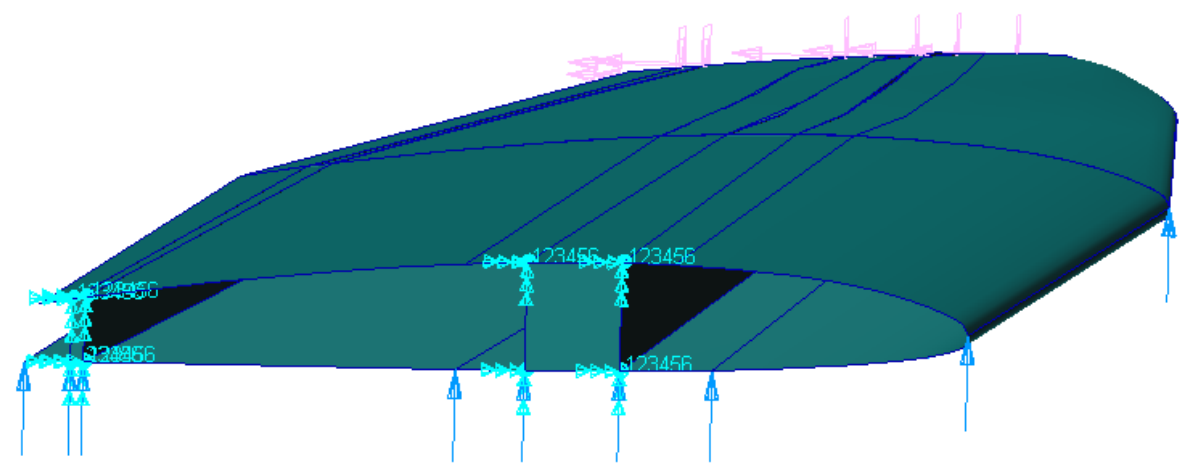

Fig. 4: Boundary conditions applied to the static structural model, cyan markers indicate fixed surface and blue arrows indicate pressure applied on a surface.

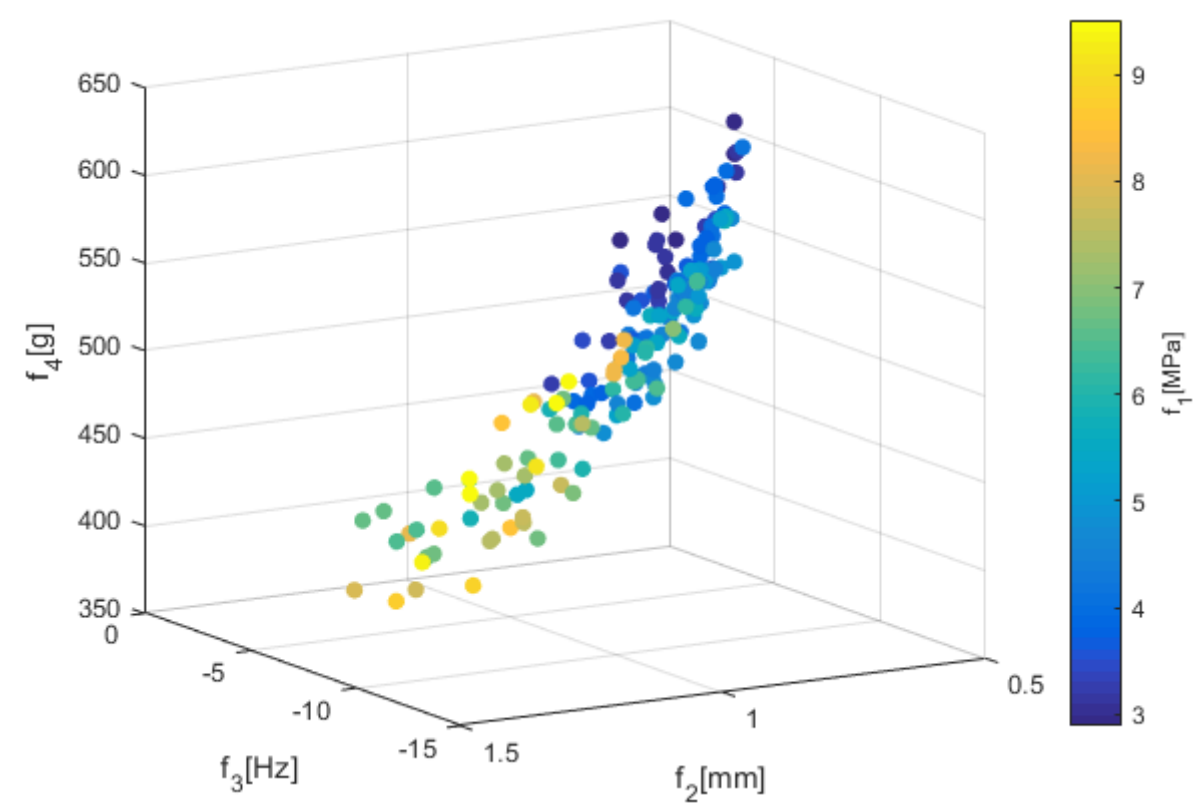

Fig. 5: 3D plot of obtained solutions.

\section{Acknowledgement}

The research is partially financed from financial resources from the statutory subsidy of the Faculty of Mechanical Engineering, Silesian University of Technology in 2020.

\section{References}

Deb, K. (1999) Multi-objective Genetic Algorithms: Problem Difficulties and Construction of Test Problems, Evolutionary Computation, 1999, vol. 7(3), pp. 205-230.

Deb, K., Pratap, A., Agarwal, S., Meyarivan, T. (2002) A fast and elitist multi-objective genetic algorithm: NSGA-II, IEEE T. Evolut. Comput, vol. 6(2), pp. 181-197.

Gay, D. (2014) Composite Materials: Design and Applications, Third Edition, 2014, CRC Press, 635 p.

Gibson, R. F. (2012) Principles of Composite Material Mechanics, 2012, CRC Press, 683 p.

Storn, R., Price, K. (1997) Differential Evolution - A Simple and Efficient Heuristic for global Optimization over Continuous Spaces. Journal of Global Optimization, vol. 11(4), pp. 341-359. 